\title{
A!
}

This is an electronic reprint of the original article.

This reprint may differ from the original in pagination and typographic detail.

Baranov, Dmitry A.; Dmitriev, Pavel A.; Mukhin, Ivan S.; Samusev, Anton K.; Belov, Pavel A.; Simovski, Constantin R.; Shalin, Alexander S.

Broadband antireflective coatings based on two-dimensional arrays of subwavelength nanopores

Published in:

Applied Physics Letters

DOI:

$10.1063 / 1.4919589$

Published: 01/01/2015

Document Version

Publisher's PDF, also known as Version of record

Please cite the original version:

Baranov, D. A., Dmitriev, P. A., Mukhin, I. S., Samusev, A. K., Belov, P. A., Simovski, C. R., \& Shalin, A. S. (2015). Broadband antireflective coatings based on two-dimensional arrays of subwavelength nanopores.

Applied Physics Letters, 106(17). https://doi.org/10.1063/1.4919589

This material is protected by copyright and other intellectual property rights, and duplication or sale of all or part of any of the repository collections is not permitted, except that material may be duplicated by you for your research use or educational purposes in electronic or print form. You must obtain permission for any other use. Electronic or print copies may not be offered, whether for sale or otherwise to anyone who is not an authorised user. 


\section{Broadband antireflective coatings based on two-dimensional arrays of subwavelength nanopores}

D. A. Baranov, P. A. Dmitriev, I. S. Mukhin, A. K. Samusev, P. A. Belov, C. R. Simovski, and A. S. Shalin

Citation: Appl. Phys. Lett. 106, 171913 (2015); doi: 10.1063/1.4919589

View online: https://doi.org/10.1063/1.4919589

View Table of Contents: http://aip.scitation.org/toc/apl/106/17

Published by the American Institute of Physics

\section{Articles you may be interested in}

Metasurface optical antireflection coating

Applied Physics Letters 105, 241113 (2014); 10.1063/1.4904827

Light-trapping and antireflective coatings for amorphous Si-based thin film solar cells

Journal of Applied Physics 117, 203101 (2015); 10.1063/1.4921440

Broadband moth-eye antireflection coatings on silicon

Applied Physics Letters 92, 061112 (2008); 10.1063/1.2870080

Wireless power transfer based on magnetic quadrupole coupling in dielectric resonators

Applied Physics Letters 108, 023902 (2016); 10.1063/1.4939789

Demonstration of the enhanced Purcell factor in all-dielectric structures

Applied Physics Letters 108, 211105 (2016); 10.1063/1.4952740

All-dielectric nanoantennas for unidirectional excitation of electromagnetic guided modes

Applied Physics Letters 107, 171101 (2015); 10.1063/1.4934757

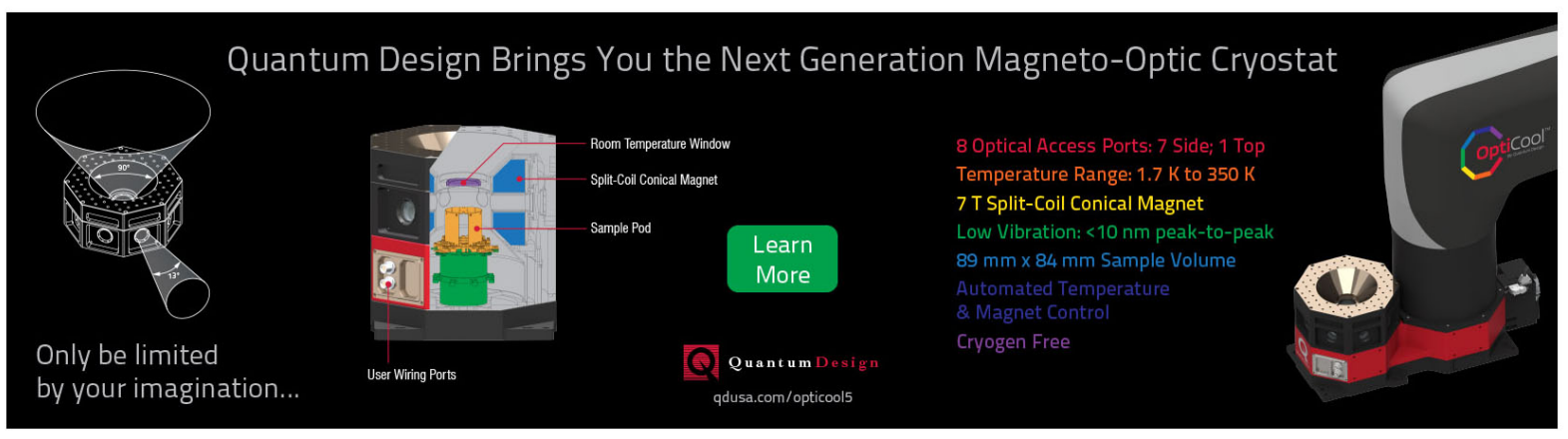




\title{
Broadband antireflective coatings based on two-dimensional arrays of subwavelength nanopores
}

\author{
D. A. Baranov, ${ }^{1}$ P. A. Dmitriev, ${ }^{1}$ I. S. Mukhin,${ }^{1,2}$ A. K. Samusev, ${ }^{1,3}$ P. A. Belov, ${ }^{1}$ \\ C. R. Simovski, ${ }^{1,4}$ and A. S. Shalin ${ }^{1,5}$ \\ ${ }^{1}$ ITMO University, St. Petersburg 197101, Russia \\ ${ }^{2}$ St. Petersburg Academic University, St. Petersburg 194012, Russia \\ ${ }^{3}$ Ioffe Physical-Technical Institute of the Russian Academy of Sciences, St. Petersburg 194021, Russia \\ ${ }^{4}$ School of Electric and Electronic Engineers, Aalto University, Aalto 00076, Finland \\ ${ }^{5}$ Ulyanovsk State University, Ulyanovsk 432017, Russia
}

(Received 10 March 2015; accepted 21 April 2015; published online 30 April 2015)

\begin{abstract}
A broadband thin-film antireflective coating (ARC) - a regular array of nanopores in a dielectric substrate-is studied theoretically and experimentally. Tuning the geometrical parameters of the array allows for strong suppression of reflection. For a fused silica substrate, reflectivity is lower than $1 \%$ over a range with a relative bandwidth, $60 \%-70 \%$, reaching $0.05 \%$ in the minimum. The underlying physics is the spatial dispersion in the porous medium which enables phase compensation for the partially reflected waves in a broad band. This allows for more broadband antireflection than any flat homogeneous single-layer ARC can provide. Importantly, the studied ARC is universal for any transparent dielectric substrate. (C) 2015 AIP Publishing LLC.
\end{abstract}

[http://dx.doi.org/10.1063/1.4919589]

At the moment, antireflective coatings (ARCs) are usually fabricated using single or multilayer homogeneous thin films. They are applicable in a wide spectral range but have a number of limitations. ${ }^{1-3}$ A single-layer flat ARC is either narrow-band or introduces losses (if a material with frequency dispersion is used to broaden the antireflection). ${ }^{1,4}$ Practically, most of the currently used single-layer ARCs do not achieve values of transmittance above $99.5 \%$ and possess dichroism. Multilayer ARCs are expensive to fabricate and are subject for degradation under thermal stress. Therefore, alternatives to homogeneous thin film ARCs have been developed recently.

Composite thin films with embedded nanoparticles extend the capabilities of single and multilayer homogeneous ARCs by supplying materials with previously unachievable properties. The film's optical properties can be tuned in a wide range by varying the concentration and type of inclusions. 5,6

Another alternative is represented by works on gradient ARCs-i.e., coatings whose effective refractive index varies from that of air on top to that of the substrate at the bottom. ${ }^{7,8}$ This can be achieved by doping homogeneous films ${ }^{9,10}$ or by texturing the surface of the substrate. ${ }^{11,12}$ In the first case, a gradient of refractive index is realized through inhomogeneous doping of the film (in a direction perpendicular to the film); in the second case, the gradient is created by nanosized protrusions grown or etched on the surface of the film (sometimes called moth-eye structures ${ }^{11}$ ).

Different metasurface-based approaches were also applied to the antireflection problem. In Refs. 13 and 14, metallic nanoantennas were used to achieve antireflection. However, due to absorption in metal, they are more common in light trapping applications ${ }^{15}$ rather than ARC. Dielectric structures, e.g., silicon nanodisk arrays ${ }^{16}$ or self-organized layers of dielectric spheres, ${ }^{17-19}$ also find their application as ARC. More complex ideas include minimizing the reflection on the 2D-photonic crystal interface ${ }^{20}$ or self-collimating photonic crystals. ${ }^{21}$

In this letter, we explore another alternative to flat ARCs - a textured thin film with unusual frequency dispersion of the phase of the reflected wave. This dispersion makes the effective optical thickness of the ARC dependent on the wavelength so that the phase shift between the waves reflected from the "vacuum-film" and "film-substrate" interfaces is maintained nearly constant in a certain spectral range. Effectively homogeneous media with such anomalous dispersion must be lossy according to the Kramers-Kronig relations. ${ }^{4}$ However, these relations lose their validity for effective non-homogeneous media with spatial dispersion. In this case, electromagnetic interaction of the constituents enables the required frequency dispersion without optical losses. However, engineering a material with such anomalous dispersion is difficult.

A possible implementation of an ARC utilizing spatial dispersion of its refractive index was introduced in Ref. 22, and in this letter, we present the experimental proof of the effect for a film textured by a regular array of cylindrical submicron pores.

Let us consider the system depicted on Fig. 1. The reflectance of such a structure can be found using the imaginary boundary method developed in Refs. 22 and 23. This method substitutes a 2D array of small inclusions with an array of point dipoles with quasi-static polarizability $\alpha_{p}$ submerged to a depth $\Delta$ inside the substrate. ${ }^{22}$

As a result, there are two boundaries in the structure: one real (substrate-free space) and one-imaginary. Mathematically, the method is expressed by an Airy-like formula for the tensor reflection coefficient $\hat{r}^{4,22}$ The details of the method are described in Refs. 22 and 23.

In the present case, the substrate is isotropic, the inclusions are cylindrical pores, and the grid is square. Moreover, 


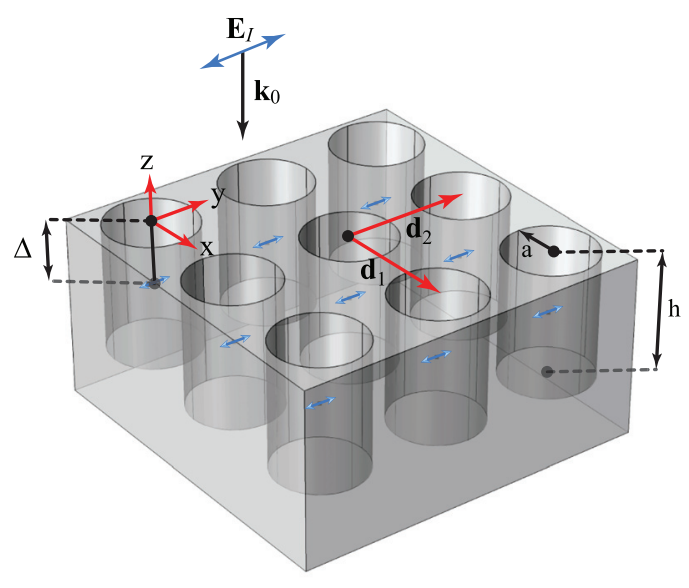

FIG. 1. ARC realized as a square lattice of submicron pores with height $h$ and radius $a$, and translation vectors $\mathbf{d}_{1}=\mathbf{d}_{2}=d$. The external electric field $\mathbf{E}_{I}$ has the wavevector $\mathbf{k}_{0}$. The distance between the surface and point-like dipole induced in each pore is $\Delta$. The refractive index of the medium is $n_{m}$.

we study only normal incidence. In this case, the reflection from the structure is canceled if and only if ${ }^{22,23}$

$$
|R|=\left|R_{l}\right|, \quad \exp \left(2 i n_{m}\left(k_{0} \Delta\right)+i \rho_{l}\right)=-1 .
$$

Here, $\left|R_{l}\right|$ is the reflection coefficient of the imaginary boundary, $\rho_{l}$ is its phase, and $|R|$ - the reflection coefficient of the "substrate-free space" boundary. Then, the amplitude and phase of the reflection coefficient $R_{l}$ can be written as $^{22,23}$

$$
\left|R_{l}\right|=\sqrt{\frac{1}{F\left(\lambda_{0}\right)^{2}+1}}, \quad \rho_{l}=\pi-\arctan \left(F\left(\lambda_{0}\right)\right),
$$

where following notations are introduced:

$$
F\left(\lambda_{0}\right) \approx \frac{\lambda_{0}}{4 \pi^{2} n_{m} d}(\kappa-4.29)+G\left(\frac{1}{\lambda_{0}}\right), \quad \kappa=\frac{d^{3}}{\alpha_{p}} .
$$

Here, $\alpha_{p}$ is the quasi-static polarizability of a single inclusion and $G\left(1 / \lambda_{0}\right)$ is a second-degree polynomial.

The reflection suppression in a broad band becomes possible when the changes of $\rho_{l}$ compensate the changes of the phase shift $2 n_{m}\left(k_{0} \Delta\right)$ with the variation of wavelength. As follows from (3), an approximate compensation of the phase shift $2 n_{m}\left(k_{0} \Delta\right)$ is possible when $n<n_{m}$, which implies the negative polarizability of an inclusion (meaning, $\alpha_{p}<0$ ). ${ }^{22,23}$ Therefore, a suitable design is pores with $n=1$.

The replacement of the inclusions (surface pores in the present work or embedded voids in Refs. 22 and 23) by point dipoles is similar to methods typical for the theory of metasurfaces (e.g., in Refs. 24 and 25). However, the concept of metasurfaces implies the homogenization of the structure. In our case, the structure is spatially dispersive because the array of small submicron pores is intentionally engineered sufficiently sparse. The spatial dispersion (i.e., nonlocality) is induced by retardation in dipole-dipole electromagnetic interaction, which enables the effect of phase compensation, see Ref. 22. Therefore, the imaginary boundary method which does not appeal to the homogenization of the array is more suitable.
In this work, the nano-patterned structures were designed and fabricated in a layer of polymethyl-methacrylate (PMMA) by electron beam lithography on a fused silica substrate. A $170 \mathrm{~nm}$ layer of PMMA was spun onto a substrate. To facilitate charge draining, a $10 \mathrm{~nm}$ layer of gold was deposited on top of the PMMA. After electron beam exposure process and before resist development, the gold layer was removed with a solution of KI. Since the refractive index of PMMA is very close to that of fused silica, the structure can be considered as an array of submicron pores formed directly in the fused silica substrate. The numerical calculations for the case of cylindrical pores were done using measured refractive indexes of fused silica and PMMA. ${ }^{26}$

The resulting samples were $20 \times 20 \mu \mathrm{m}^{2}$ square arrays of submicron pores with periods of 250,300 , and $200 \mathrm{~nm}$ (samples \#1, \#2, and \#3, respectively) with fill-factor (i.e., ratio between volume of the pore and volume of the unit cell) ranged from $40 \%$ to $80 \%$. The topography of the resulting nanostructured ARC including the thickness of the PMMA layer was characterized by atomic force microscopy (AFM). Typical AFM images are shown in Figs. 2(a) and 2(b). Pore depths measured by AFM are equal to $120 \mathrm{~nm}$ and $135 \mathrm{~nm}$ for samples \#1 and \#2, respectively. The lateral dimensions of the pores are slightly distorted due to the shape of the AFM cantilever tip which reduces the slope angle and resolution. ${ }^{27}$ The actual thickness of the nanopore arrays is lower than the thickness of the PMMA layer because of parasitic exposure and development of the PMMA around the pores due to proximity effect. Typical cross-sections of the pores in samples \#1 and \#2 are shown in Figs. 2(c) and 2(d), respectively.

The optical spectroscopy experimental setup is depicted in Fig. 3. Reflection spectra were obtained using confocal microspectroscopy ${ }^{28}$ in the visible range $(400-760 \mathrm{~nm})$. To avoid parasitic reflection from the back face of the substrate, the bottom interface was immersed in an index matching liquid. The reference reflection was obtained using an aluminium mirror with a known reflection spectrum.

The main challenge in measuring the close-to-zero reflection spectra is accurate normalization to obtain a reliable absolute value of reflectivity. The achieved absolute error did not exceed $0.1 \%$ reflectivity, ensuring the reliability of the experimental spectra. The accuracy of the experiment was additionally verified by comparing the theoretical and experimental reflectivities of the fused silica substrate (see Fig. 4). The discrepancy between the theoretical and experimental values is of the same order as the discrepancy of different theoretical models of the reflection from a multilayer structure (we used the finite-difference time-domain method (FDTD, Lumerical $^{29}$ and the Transfer Matrix Method ${ }^{4}$ ). Since we immersed the back face of the sample, our simulations were carried out in the approximation of a semi-infinite substrate.

The theoretical and experimental reflection spectra of virgin fused silica (top curve) and the samples (bottom curves) are shown in Fig. 4. Reflection from the samples is almost completely suppressed at the central wavelength and is lower than $1 \%$ in a wide band (which for sample \#2 is designed to cover the visible range). For sample \#1, the reflection in the $400-600 \mathrm{~nm}$ spectral range is reduced to nearly $0.5 \%$. The comparison of data for two samples 

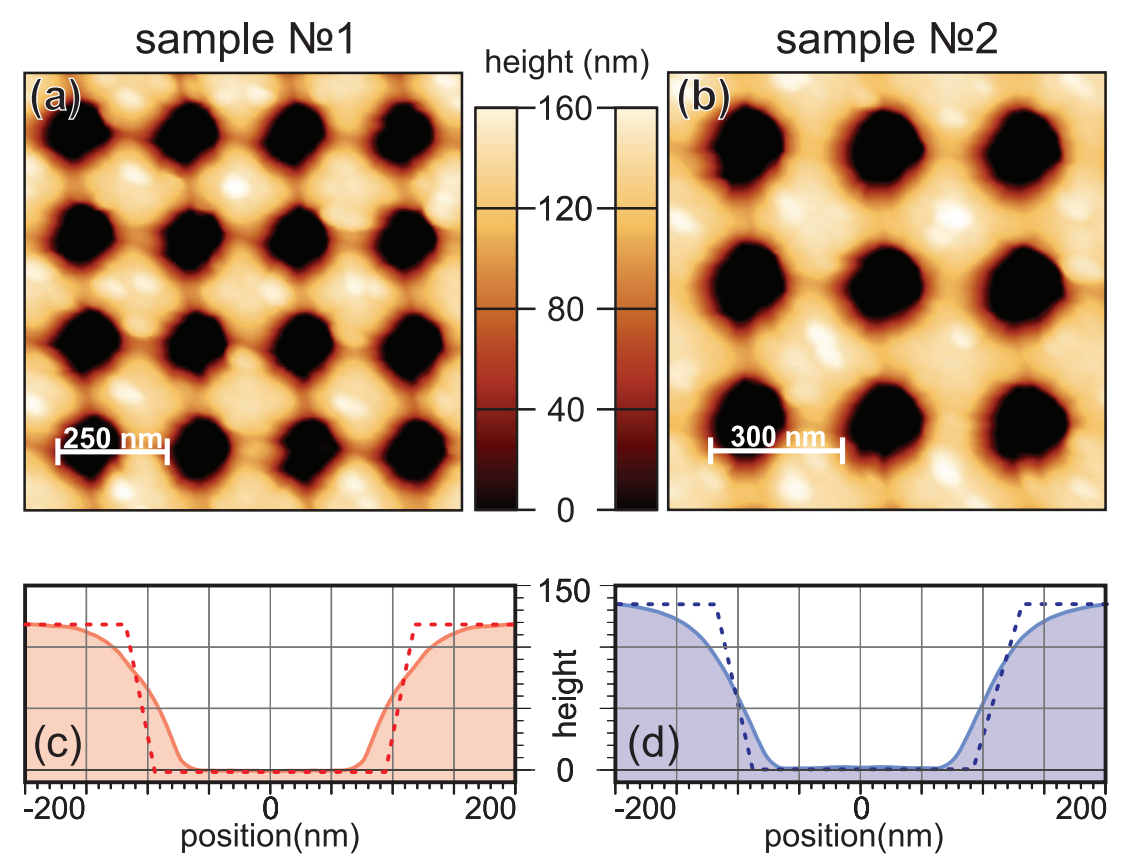

FIG. 2. Atomic force microscopy images of nanoporous antireflective coating samples (a) and (b). The crosssections of single pores (filled areas) and cone dimensions corresponding to the best matching FDTD fits (dashed line) (c) and (d).

illustrates the impact of geometrical parameters of the array on the position of the reflection minimum, whereas the relative bandwidth is virtually constant. The discrepancy between the experimental and simulated spectra for sample \#2 at wavelengths shorter than $460 \mathrm{~nm}$ can be explained by the contribution of diffraction on the array (which has a period of $300 \mathrm{~nm}$ ). The red edge of the diffraction band is shown by a vertical line. For sample \#1, this edge is below $400 \mathrm{~nm}$.

In Fig. 4, we also show the reflection spectrum for a quarter-wave antireflection coating (black line) designed so as to suppress the reflection at the same wavelength as sample \#1 does. The frequency dependence of the reflectivity granted by the $\lambda / 4 \mathrm{ARC}$ is nearly the same as that offered by our ARC. The $\lambda / 4$ layer has an optical thickness of $D=\lambda_{0} / 4 n_{A R C} \approx 94.14 \mathrm{~nm}$ at $\lambda_{0}=470 \mathrm{~nm}$ and a matched refractive index $n_{A R C}=1.244$, which nearly satisfies the

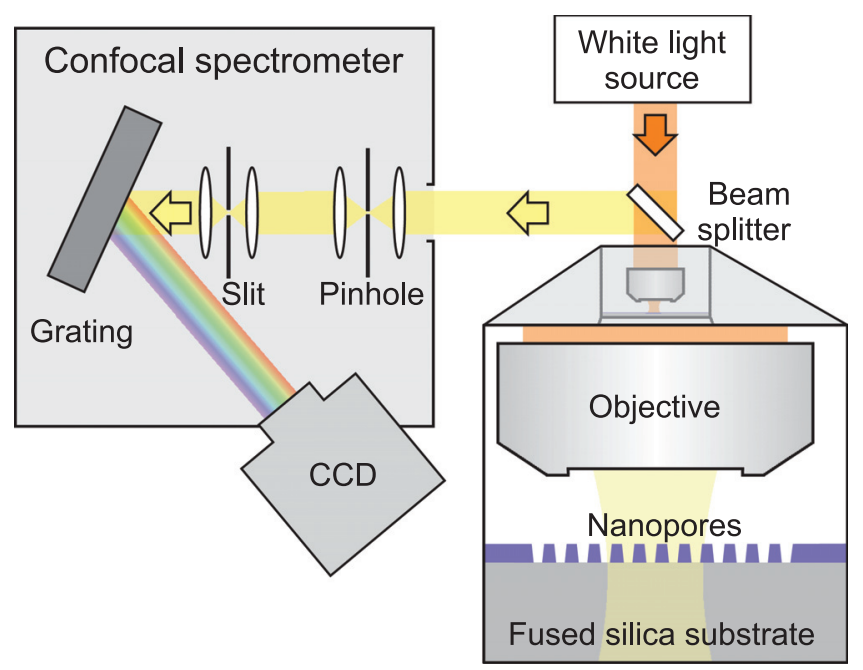

FIG. 3. Optical spectroscopy experimental setup. The sample is illuminated using a HL-2000 FHSA tungsten halogen light source through a Mitutoyo M Plan Apo $50 \times / 0.55$ objective lens. The reflected light is collected by the same objective and passed to a confocal spectrometer. The confocal pinhole size is $150 \mu \mathrm{m}$ which corresponds to a $10 \times 10 \mu \mathrm{m}^{2}$ collection area. second condition of antireflection $\left(n_{A R C}=\sqrt{n_{m}}\right) .{ }^{4}$ The material of the $\lambda / 4-\mathrm{ARC}$ is an abstract medium, whose refractive index $n=1.244$ would be difficult to synthesize with needed precision. Therefore, our ARC mimics an idealized single-layer ARC and any single-layer ARC of available materials will provide worse antireflection.

However, our ARC should theoretically work even better than any $\lambda / 4$-coating due to the broadband phase compensation. $^{22}$ According to formulas (2 and 3), in order to achieve the phase compensation in a broad band, one needs a high absolute value for the parameter $\kappa=d^{3} / \alpha_{p}=-d^{3} /\left|\alpha_{p}\right|$. To decrease $\alpha_{p}$ or to increase $d$ implies decreasing of the fillfactor of the nanopores. In this case, the reflectance $\left|R_{l}\right|$ from the imaginary boundary decreases and becomes smaller than $|R|$, the reflectance of the pure substrate, which leads to higher values of reflection minimum. So, it is difficult to combine both phase and amplitude conditions for the complete reflection suppression in wide band and one needs to accurately tune the geometrical parameters of the nanopores.

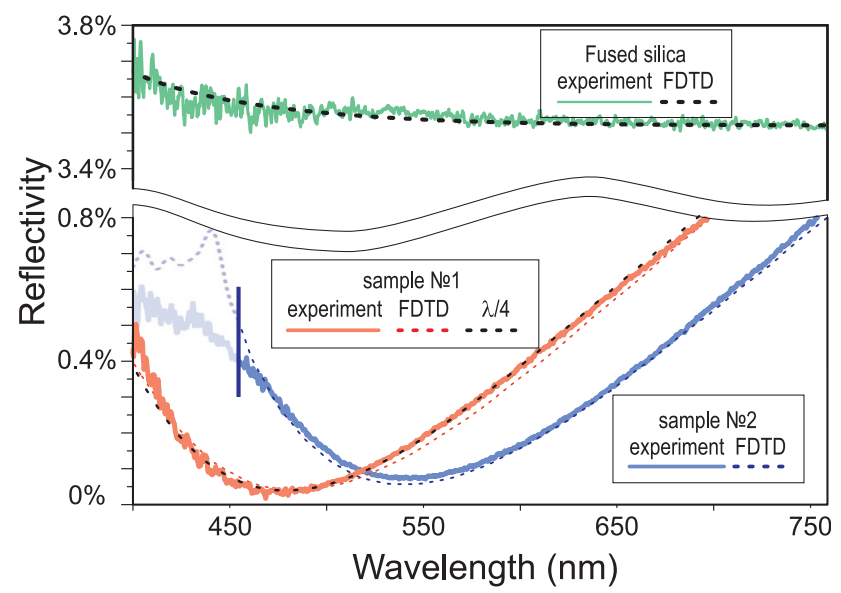

FIG. 4. Experimental (solid lines) and numerically simulated (dashed lines) reflection spectra for pure fused silica substrate and two samples. For sample $\# 1$ (red line), matching $\lambda / 4(\mathrm{n}=1.244, \mathrm{D}=94.14 \mathrm{~nm})$ coating is shown by black dotted line. 


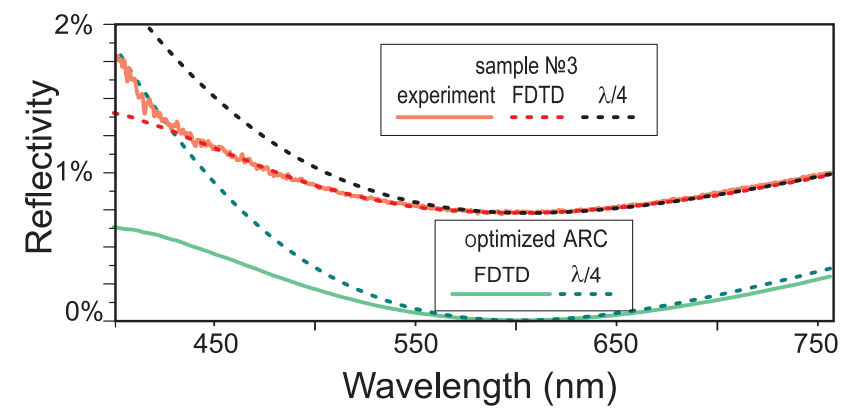

FIG. 5. Experimental (red solid line) and simulated (red dashed line) reflection spectra and matching $\lambda / 4(\mathrm{n}=1.171, \mathrm{D}=127.5)$ coating (black dashed line). Green lines show the optimal reflection spectra (solid line) calculated by FDTD and respective $\lambda / 4(\mathrm{n}=1.236, \mathrm{D}=121.32)$ coating (dashed line).

Figure 5 shows reflection spectra for sample \#3: FDTD model, experiment, and a matching $\lambda / 4$-ARC having the same minimal reflectivity at the same wavelength $(600 \mathrm{~nm})$. In this case, our ARC is more broadband than the $\lambda / 4$-ARC, but the reflection minimum value is about $0.9 \%$. This is because of high values of $\kappa$ and effective phase compensation as was shown before.

Phase compensation and the spectral interval of antireflection can be further increased by optimization of the pore shape. In Figure 5, we also show the reflection spectrum offered by FDTD simulations for an optimized shape and compare this curve with the reflectivity of a single-layer ARC with minimal reflectance at the same wavelength (shown green). Here, the contrast in the operation bandwidth in favor of our ARC is more expressed. We can therefore conclude that a more elaborate comparison of our ARC with single-layer ARCs can show the advantages of our structure, namely, in the broader operation band.

Another advantage of the proposed ARC is its universality. Reference 30 shows, for the case of spherical voids, that reflection spectra qualitatively similar to those shown in Figs. 4 and 5 are possible for any transparent dielectric. This is because the variation of the parameters of pores and their arrangement significantly changes the optical properties of the ARC, making it adjustable for various substrates.

To conclude, we have proved the viability of submicron pore arrays as very efficient and broadband ARCs through both theoretical and experimental studies. Our ARC works better than any single layer homogeneous ARC and has two advantages: it can be fabricated directly in the substrate and is more broadband. The mechanism of broadband antireflection is phase compensation caused by spatial dispersion rather than a gradient of refractive index.
This work has been partially supported by Government of the Russian Federation (Grant No. 074-U01), the support of the Ministry of Education and Science of the Russian Federation (GOSZADANIE 2014/190, Project No. 14.Z50.31.0015), and the Russian Foundation for Basic Research (Grant Nos. 14-0831730 mol-a and 14-02-31765 mol-a).

${ }^{1}$ H. K. Raut, V. A. Ganesh, A. S. Nair, and S. Ramakrishna, Energy Environ. Sci. 4, 3779 (2011).

${ }^{2}$ S. Walheim, E. Schäffer, J. Mlynek, and U. Steiner, Science 283, 520 (1999).

${ }^{3}$ P. Lalanne and G. M. Morris, Nanotechnology 8, 53 (1997).

${ }^{4}$ M. Born and E. Wolf, Principles of Optics: Electromagnetic Theory of Propagation, Interference and Diffraction of Light (CUP Archive, 1999).

${ }^{5}$ K. C. Krogman, T. Druffel, and M. K. Sunkara, Nanotechnology 16, S338 (2005).

${ }^{6}$ J. Xiong, S. N. Das, J. P. Kar, J.-H. Choi, and J.-M. Myoung, J. Mater. Chem. 20, 10246 (2010).

${ }^{7}$ L. Rayleigh, Proc. London Math. Soc. 1, 51 (1879).

${ }^{8}$ W. H. Southwell, Opt. Lett. 8, 584 (1983).

${ }^{9}$ W. Qiu, Y. Ma, J. Zhao, J.-X. Wang, M. Li, S. Li, and J. Pan, Jpn. J. Appl. Phys., Part 1 53, 021501 (2014).

${ }^{10}$ J. W. Leem, Y. P. Kim, and J. S. Yu, J. Opt. Soc. Am. B 29, 357 (2012).

${ }^{11}$ L. Wang, B.-B. Xu, Q.-D. Chen, Z.-C. Ma, R. Zhang, Q.-X. Liu, and H.-B. Sun, Opt. Lett. 36, 3305 (2011).

${ }^{12}$ Y. Kanamori, M. Sasaki, and K. Hane, Opt. Lett. 24, 1422 (1999).

${ }^{13}$ H.-T. Chen, J. Zhou, J. F. O'Hara, F. Chen, A. K. Azad, and A. J. Taylor, Phys. Rev. Lett. 105, 073901 (2010).

${ }^{14}$ B. Zhang, J. Hendrickson, N. Nader, H.-T. Chen, and J. Guo, Appl. Phys. Lett. 105, 241113 (2014).

${ }^{15}$ C. R. Simovski, A. S. Shalin, P. M. Voroshilov, and P. A. Belov, J. Appl. Phys. 114, 103104 (2013).

${ }^{16}$ I. Staude, A. E. Miroshnichenko, M. Decker, N. T. Fofang, S. Liu, E. Gonzales, J. Dominguez, T. S. Luk, D. N. Neshev, I. Brener et al., ACS Nano 7, 7824 (2013).

${ }^{17}$ B. G. Prevo, E. W. Hon, and O. D. Velev, J. Mater. Chem. 17, 791 (2007).

${ }^{18}$ Y. Wang, L. Chen, H. Yang, Q. Guo, W. Zhou, and M. Tao, Sol. Energy Mater. Sol. Cells 93, 85 (2009).

${ }^{19}$ C.-H. Chan, A. Fischer, A. Martinez-Gil, P. Taillepierre, C.-C. Lee, S.-L. Yang, C.-H. Hou, H.-T. Chien, D.-P. Cai, K.-C. Hsu et al., Appl. Phys. B 100, 547 (2010).

${ }^{20}$ S.-G. Lee, J.-s. Choi, J.-E. Kim, H.-Y. Park, and C.-S. Kee, Opt. Express 16, 4270 (2008).

${ }^{21}$ J.-M. Park, S.-G. Lee, H.-R. Park, and M.-H. Lee, Opt. Express 18, 13083 (2010).

${ }^{22}$ A. S. Shalin, JETP Lett. 91, 636 (2010).

${ }^{23}$ A. S. Shalin, Quantum Electron. 41, 163 (2011).

${ }^{24}$ M. Albooyeh, D. Morits, and C. Simovski, Metamaterials 5, 178 (2011).

${ }^{25}$ C. L. Holloway, A. Dienstfrey, E. F. Kuester, J. F. O’Hara, A. K. Azad, and A. J. Taylor, Metamaterials 3, 100 (2009).

${ }^{26}$ See http://refractiveindex.info/ for Refractive index database.

${ }^{27}$ P. Markiewicz and M. C. Goh, Langmuir 10, 5 (1994).

${ }^{28}$ M. J. Booth, R. Juškaitis, and T. Wilson, J. Eur. Opt. Soc. 3, 08026 (2008).

${ }^{29} \mathrm{See} \quad \mathrm{http} / / / \mathrm{www}$.lumerical.com/tcad-products/fdtd/ for Lumerical Solutions, Inc.

${ }^{30}$ A. S. Shalin and S. A. Nikitov, Prog. Electromagn. Res. B 47, 127 (2013). 